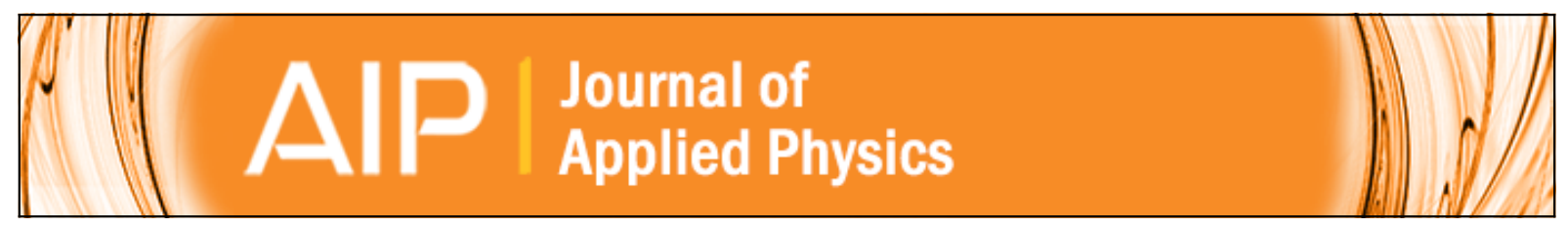

\title{
In situ laser-based resonant ultrasound measurements of microstructure mediated mechanical property evolution
}

D. H. Hurley, S. J. Reese, S. K. Park, Z. Utegulov, J. R. Kennedy, and K. L. Telschow

Citation: Journal of Applied Physics 107, 063510 (2010); doi: 10.1063/1.3327428

View online: http://dx.doi.org/10.1063/1.3327428

View Table of Contents: http://scitation.aip.org/content/aip/journal/jap/107/6?ver=pdfcov

Published by the AIP Publishing

\section{Articles you may be interested in}

Selective laser melting of aluminum die-cast alloy—Correlations between process parameters, solidification conditions, and resulting mechanical properties

J. Laser Appl. 27, S29205 (2015); 10.2351/1.4906389

Optical fiber technique for in-reactor mechanical properties measurement

AIP Conf. Proc. 1511, 1701 (2013); 10.1063/1.4789246

Application of laser-based resonant ultrasound spectroscopy to study texture in copper

J. Appl. Phys. 111, 053527 (2012); 10.1063/1.3692386

Evolution of magnetic properties and crystallographic texture in electrical steel with large plastic deformation J. Appl. Phys. 109, 07 A325 (2011); 10.1063/1.3560895

Microstructure and mechanical property characterizations of metal foil after microscale laser dynamic forming J. Appl. Phys. 101, 063108 (2007); 10.1063/1.2710334

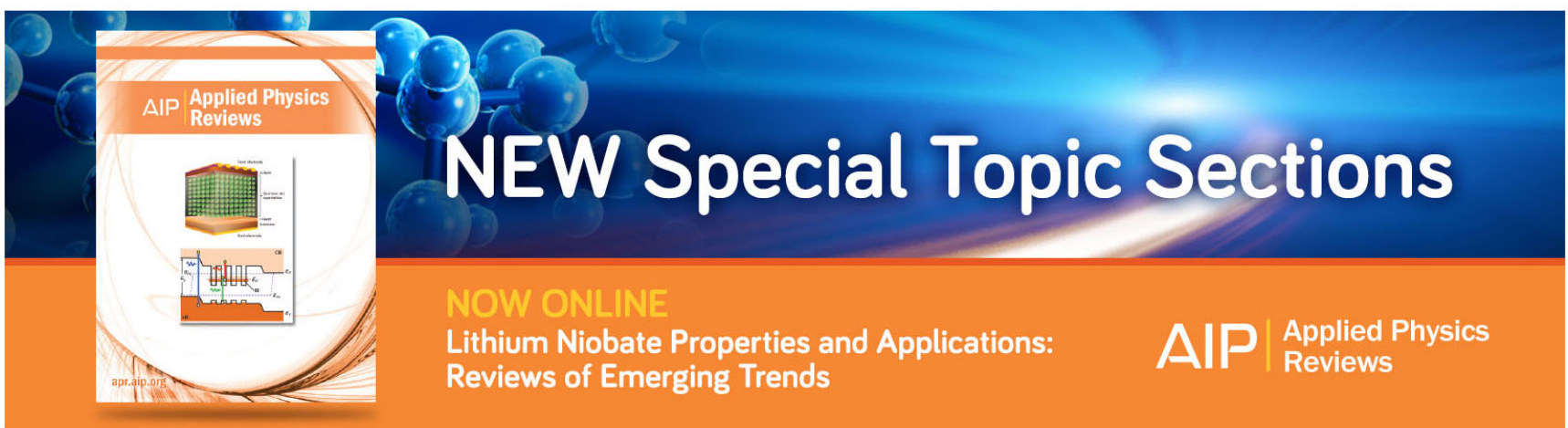




\title{
In situ laser-based resonant ultrasound measurements of microstructure mediated mechanical property evolution
}

\author{
D. H. Hurley, ${ }^{1, a)}$ S. J. Reese, ${ }^{1}$ S. K. Park, ${ }^{2}$ Z. Utegulov, ${ }^{1}$ J. R. Kennedy, ${ }^{1}$ and \\ K. L. Telschow ${ }^{1}$ \\ ${ }^{1}$ Idaho National Laboratory, Idaho Falls, Idaho 83415, USA \\ ${ }^{2}$ Korean Atomic Energy Research Institute, Daejeon 305-353, South Korea
}

(Received 1 October 2009; accepted 24 January 2010; published online 24 March 2010)

\begin{abstract}
In situ laser-based resonant ultrasound spectroscopy is used to characterize the development of a recrystallized microstructure in a high purity copper sample. The modal shapes, used for mode identification, of several resonant modes are determined before and after annealing by raster scanning the laser interferometric probe. This information is used to isolate the motion of individual modes during high temperature annealing. The evolution of a particular mode during annealing is examined in detail. During recrystallization, the center frequency of this mode shifts by approximately $20 \%$ of the original value. Using electron backscatter data it is shown that the majority of this shift is due to changes in the polycrystal average elastic stiffness tensor, driven by changes in texture, and that changes in dislocation density and pinning length are secondary influences. (C) 2010 American Institute of Physics. [doi:10.1063/1.3327428]
\end{abstract}

\section{INTRODUCTION}

Resonant ultrasound spectroscopy (RUS) is an effective tool to measure mechanical properties of materials. ${ }^{1-4}$ The resonant response, as determined by the elastic constants and ultrasonic dissipation, has been shown to be a strong function of the microstructural state of the material. Much of the previous work in this area has focused on relating changes in the resonant response to changes in dislocation density and pinning length. This approach ${ }^{5,6}$ involves dividing the modulus into a purely elastic modulus and a dislocation modulus. Because an ultrasonic wave transfers energy to a dislocation by causing it to oscillate, the dislocation modulus is necessarily complex with the real part associated with changes in acoustic velocity and the imaginary part associated with changes in ultrasonic attenuation. The work of Thompson and Holmes ${ }^{7}$ provides one of the first examples of using RUS to monitor in situ changes in mechanical properties. Their results, involving high purity copper samples exposed to neutron radiation, showed a marked increase in Young's modulus and a decrease in attenuation with increasing radiation dose. Using the oscillating-dislocation-line model of Granato and Lucke ${ }^{8,9}$ to interpret their results, they showed that the change in modulus and attenuation could be related to dislocation pinning by radiation-induced defects. Many other studies ${ }^{10-13}$ have confirmed the basic elements of this explanation; however, wide disagreement between experimental results has precluded specification of the type and size of the defect responsible for pinning. At the time of these studies this limitation was likely due to uncertainty in the initial and final state of the microstructure. ${ }^{14}$

A related area of investigation involves monitoring mechanical property evolution driven by changes in temperature. ${ }^{4,15-19}$ This approach is necessarily more complicated than isothermal irradiation studies in that it can also

${ }^{a)}$ Electronic mail: david.hurley@inl.gov. entail changes in grain texture. For investigations involving the annealing of heavily cold worked metals, many research groups have noted a step change in the elastic modulus and an irreversible peak in ultrasonic attenuation (internal friction) as a function of increasing temperature. There is still much debate over the mechanism responsible for these changes in the resonant spectrum. ${ }^{15}$ For high purity copper $(\mathrm{Cu})$ it is thought that dislocations play a key role. ${ }^{16}$ Gondi and Tognato ${ }^{17}$ proposed a mechanism governed by dislocation climb. Subsequent work by Akhmadeev et al. ${ }^{18}$ involving the recrystallization of samples subjected to equal channel angular extrusion demonstrated that the temperature at which the internal friction peak occurs is independent of frequency. This result is inconsistent with the dislocation climb framework. More recently, Lee and Okuda ${ }^{19}$ have measured the internal friction and dynamic modulus of copper samples using a resonant flexural vibration technique. Utilizing x-ray characterization, they noted the important influence of texture on the change in dynamic modulus.

Indeed, recrystallization can involve pronounced changes in texture in addition to changes in dislocation density and pinning length. For polycrystalline materials that have large single crystal elastic anisotropy, texture and texture evolution can have a significant influence on the resonant spectrum. ${ }^{20}$ In this study we use laser-based resonant ultrasound spectroscopy (LRUS) ${ }^{21-23}$ in combination with electron backscatter diffraction (EBD) to gauge the influence of texture evolution on the resonant spectrum of a deformed polycrystalline $\mathrm{Cu}$ sample during high temperature annealing. A pulsed laser is used to locally excite a broad spectrum of resonant modes. A laser interferometer is raster scanned once prior to annealing and once after annealing to form an image of each resonant mode in the rolled and annealed states. This information, used for mode identification, enables unambiguous tracking of individual modes during high temperature annealing. Utilizing a theory that connects mode 


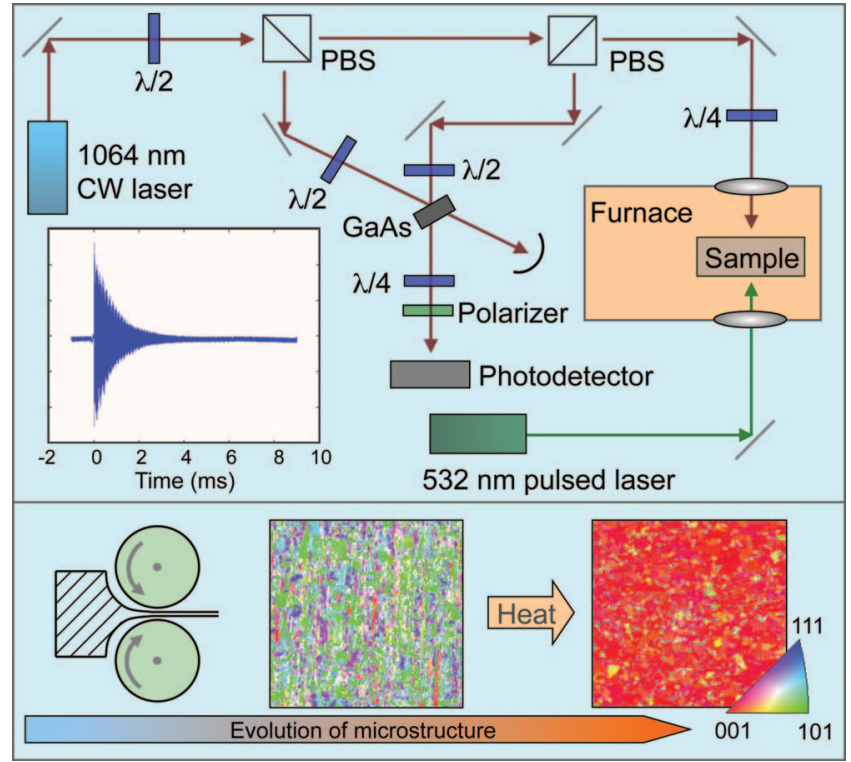

FIG. 1. (Color) Top: Experimental setup. A pulsed Nd:YAG laser is used for ultrasonic generation and a photorefractive interferometer is used to detect out-of-plane surface motion. The inset in the bottom left shows the ultrasonic ring-down in the sample after laser excitation. Bottom: Details of microstructure evolution. A texture is imparted by rolling. A well defined cube texture is formed upon annealing.

shape to the polycrystal average elastic stiffness tensor (derived from EBD data), it is demonstrated that this approach has the potential to differentiate dislocation and texture driven changes in the elastic modulus.

\section{EXPERIMENT}

The experimental setup used for ultrasonic generation and detection is shown in the top pane of Fig. 1. Broadband resonant ultrasonic modes are thermoelastically excited in a sample using a frequency doubled, pulsed neodymium-doped yttrium aluminum garnet $(\mathrm{Nd}: \mathrm{YAG})$ laser operating at 532 $\mathrm{nm}$ and a pulse duration of $10 \mathrm{~ns}$. A photorefractive interferometer is used to detect ultrasound by demodulating changes in optical phases associated with out-of-plane surface motion. Aspects of the interferometer are described in detail by Ing and Monchalin, ${ }^{24}$ Pouet et al. ${ }^{25}$ and Lafond et al. ${ }^{26}$ The interferometer is comprised of a $1064 \mathrm{~nm} \mathrm{cw}$ laser split into reference and signal beams which recombine and interfere in a gallium arsenide (GaAs) crystal. $^{27}$

The samples investigated consist of high purity $(5 \mathrm{~N}) \mathrm{Cu}$ parallelepipeds with nominal dimensions $5.4 \times 3.4$ $\times 0.54 \mathrm{~mm}$. A textured microstructure was imparted by rolling a fully annealed sample. A $97.5 \%$ reduction was produced by rolling the material many times. Upon annealing, a strong texture develops that is almost completely cubically aligned. $^{28}$ The bottom pane of Fig. 1 shows the electron backscatter micrographs of the rolled and annealed microstructures. It is noted that the cube texture (red in the micrographs) is a finite but very small part of the rolled texture, ${ }^{29}$ however, the dominate grain orientation of the rolled and recrystallized microstructures are very different. Thus, because single crystal $\mathrm{Cu}$ is highly elastically anisotropic, tex-

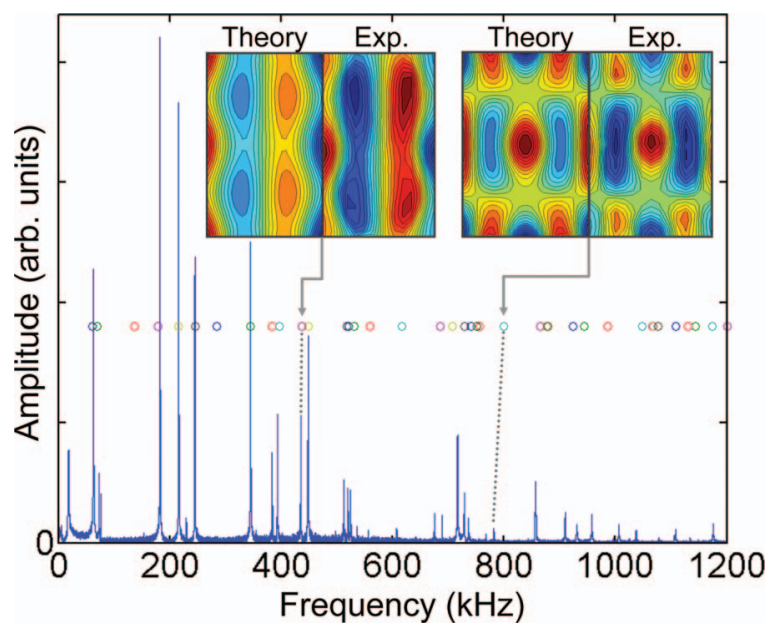

FIG. 2. (Color) The resonant spectrum of the rolled sample. Experimentally measured and predicted mode shapes are compared in the inset.

ture evolution upon annealing provides an excellent test case to investigate the influence of texture evolution on the resonant spectrum.

The generation beam strikes the sample at one corner of a $5.4 \times 3.4 \mathrm{~mm}$ face, and the detection beam measures the response from the opposed polished face. For investigations conducted before and after annealing, the mode shapes of the individual modes are formed by raster scanning the probe beam across the rectangular sample surface. For each probe position, the temporal waveform of the resonant ring down was averaged 100 times (see inset in top pane of Fig. 1). The response was measured on a grid of 375 points, with the step between points being approximately $220 \mu \mathrm{m}$. For in situ investigations during annealing, the generation and detection laser beams irradiate opposite sides of the sample which resides inside a tube furnace. In order to monitor relatively fast changes in microstructure during annealing (approximately $30 \mathrm{~s}$ intervals) the probe beam is not raster scanned. To prevent oxidation, the furnace is fitted with optical windows and purged with nitrogen gas (Fig. 1).

\section{RESULTS AND DISCUSSION}

The resonant spectrum corresponding to the rolled sample is shown in Fig. 2. Experimentally measured and predicted mode shapes are shown in the inset. The experimental mode shapes are constructed by first scanning the probe beam to acquire a 375-point array of out-of-plane displacement waveforms. The frequency domain response is obtained by taking the Fourier transform of each displacement waveform. For a resonant mode of interest, the data are then windowed in the frequency domain and the amplitude and phase are recorded. This process enables the creation of contour plots of the out-of-plane surface motion (i.e., mode shape). The predicted mode shapes were obtained using the XYZ algorithm of Visscher et al. ${ }^{2}$ The macroscopic elastic constants used as input were obtained by forming a polycrystal average elastic stiffness tensor using the Voigt-ReussHill formalism and EBD data. ${ }^{30,31,23}$ The EBD data consist of a set of Euler angles that define the crystallographic orientation of each pixel. Because the scanned area is much larger 
TABLE I. Comparison between measured and predicted mode frequencies.

\begin{tabular}{|c|c|c|c|c|c|}
\hline \multirow[b]{2}{*}{$\begin{array}{c}\Delta \mathrm{f} \\
(\%)\end{array}$} & \multicolumn{2}{|c|}{ Rolled } & \multicolumn{2}{|c|}{ Annealed } & \multirow[b]{2}{*}{$\begin{array}{l}\Delta \mathrm{f} \\
(\%)\end{array}$} \\
\hline & $\begin{array}{l}\text { Experiment } \\
(\mathrm{kHz})\end{array}$ & $\begin{array}{l}\text { Theory } \\
(\mathrm{kHz})\end{array}$ & $\begin{array}{l}\text { Theory } \\
(\mathrm{kHz})\end{array}$ & $\begin{array}{c}\text { Experiment } \\
(\mathrm{kHz})\end{array}$ & \\
\hline 4.39 & 63.404 & 60.735 & 74.331 & 81.308 & 9.39 \\
\hline \multirow[t]{2}{*}{4.27} & 73.432 & 70.424 & 53.931 & 54.680 & 1.39 \\
\hline & & 137.116 & 155.430 & 165.027 & 6.17 \\
\hline 1.90 & 182.077 & 178.689 & 139.111 & 141.457 & 1.69 \\
\hline 1.75 & 182.077 & 178.937 & 145.992 & 143.463 & 1.73 \\
\hline 0.15 & 216.271 & 215.941 & 198.207 & 205.555 & 3.71 \\
\hline 0.10 & 245.068 & 245.320 & 252.642 & 262.008 & 3.71 \\
\hline 0.04 & 284.935 & 285.051 & 313.158 & 327.087 & 4.45 \\
\hline 0.01 & 345.286 & 345.258 & 280.592 & 276.984 & 1.29 \\
\hline 0.06 & 384.019 & 383.772 & 384.681 & 391.654 & 1.81 \\
\hline \multirow[t]{2}{*}{0.92} & 393.341 & 396.989 & & & \\
\hline & & & 434.402 & 436.329 & 0.44 \\
\hline 0.37 & 435.120 & 436.719 & 347.673 & 353.474 & 1.67 \\
\hline \multirow[t]{2}{*}{0.09} & 448.346 & 448.759 & 394.632 & 404.188 & 2.42 \\
\hline & & & 452.366 & & \\
\hline 1.06 & 512.459 & 517.960 & 508.679 & 526.980 & 3.60 \\
\hline 0.21 & 520.062 & 521.172 & & & \\
\hline 1.29 & 524.849 & 531.725 & 577.693 & & \\
\hline \multirow[t]{2}{*}{0.58} & 556.058 & 559.329 & 529.557 & 533.345 & 0.72 \\
\hline & & 617.615 & 652.272 & 679.328 & 4.15 \\
\hline \multirow[t]{2}{*}{1.58} & 675.075 & 685.888 & 736.581 & 757.166 & 2.79 \\
\hline & & 707.036 & 622.341 & & \\
\hline 1.73 & 716.646 & 729.296 & 810.228 & 842.211 & 3.95 \\
\hline 1.72 & 728.738 & 741.480 & 653.234 & 658.563 & 0.82 \\
\hline 1.95 & 736.242 & 750.910 & 664.301 & 675.407 & 1.67 \\
\hline 1.30 & 745.789 & 755.595 & 703.476 & 700.289 & 0.45 \\
\hline \multirow[t]{2}{*}{2.19} & 781.916 & 799.415 & 762.566 & 781.923 & 2.54 \\
\hline & & 865.458 & 899.753 & 917.097 & 1.93 \\
\hline \multirow[t]{2}{*}{2.25} & 857.128 & 876.841 & 965.277 & 1021.216 & 5.80 \\
\hline & & 878.552 & 899.929 & 925.031 & 2.79 \\
\hline 1.46 & 909.800 & 923.305 & 839.413 & 823.326 & 1.92 \\
\hline \multirow[t]{2}{*}{1.37} & 930.367 & 943.268 & 891.901 & 883.001 & 1.00 \\
\hline & & & 968.277 & 979.694 & 1.18 \\
\hline 2.94 & 957.632 & 986.658 & & & \\
\hline 4.07 & 1006.186 & 1048.920 & 1000.821 & 992.002 & 0.88 \\
\hline 1.47 & \multicolumn{4}{|c|}{ Average } & 2.62 \\
\hline
\end{tabular}

than the average grain size, the EBD data are representative of the overall microstructure. ${ }^{32} \mathrm{~A}$ false color map of the grain orientations is shown in the bottom pane of Fig. 1. The polycrystal stiffness (Voigt) and compliance (Reuss) tensors are formed by rotating the single crystal stiffness and compliance tensors for each orientation. The resulting tensors are averaged. The Voigt-Reuss-Hill stiffness tensor is obtained by taking a geometric average of the Voigt and Reuss stiffness tensors.

In Fig. 2, a dashed line connects the predicted mode location (open circles) to the measured mode position. The same analysis was performed after the sample was annealed. The measured and predicted mode locations for the rolled and annealed states are tabulated in Table I. For the rolled sample 27 modes were identified and the average difference between theory and experiment was less than $1.5 \%$. For the annealed sample 29 modes were identified and the average difference between theory and experiment was less than $2.7 \%$. The close agreement between experiment and predic- tion confirms that the EBD data are representative of the bulk microstructure and confirm the validity of the averaging routine used to obtain the polycrystal elastic stiffness tensor. Table I reveals that some modes shift a considerable fraction of their original center frequency. Because copper has a large single crystal elastic anisotropy, the polycrystal elastic properties change dramatically as the microstructure transforms from a rolled texture to a recrystallized texture.

The texture evolution can also be examined by considering the elastic anisotropy derived from the average elastic stiffness tensor. The averaged elastic stiffness tensors for the rolled and annealed states, given in the Appendix, have essentially the same form as a single crystal material having cubic symmetry. Averaging these values [i.e., $\left(C_{11}+C_{22}\right.$ $\left.+C_{33}\right) / 3=\hat{C}_{11}, \quad\left(C_{12}+C_{13}+C_{23}\right) / 3=\hat{C}_{12}, \quad$ and $\quad\left(C_{44}+C_{55}\right.$ $\left.\left.+C_{66}\right) / 3=\hat{C}_{44}\right]$ enables calculation of the Zener anisotropy factor, ${ }^{33} A=2 \hat{C}_{44} /\left(\hat{C}_{11}-\hat{C}_{12}\right)$. For the rolled and annealed samples, $A=0.8$ and 2.4 , respectively. A sample with ran- 


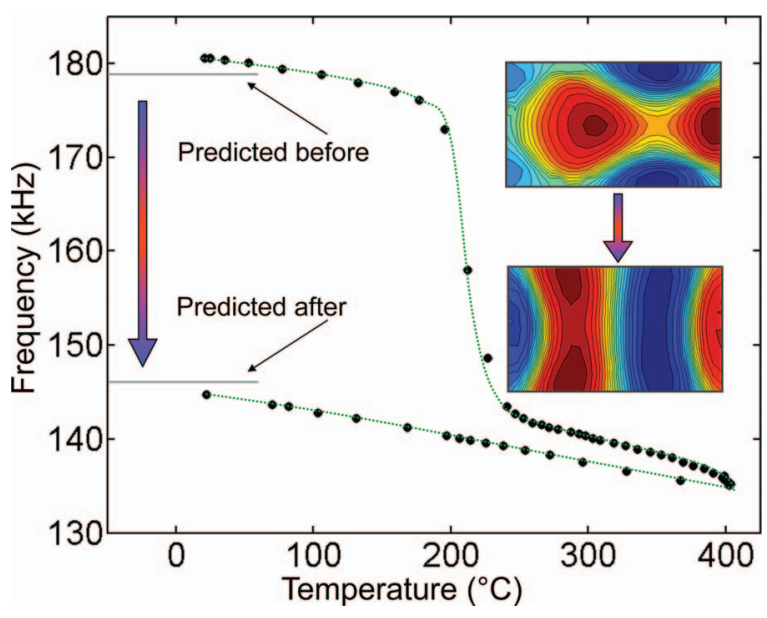

FIG. 3. (Color) In situ monitoring of resonant peak location as a function of annealing temperature. The center frequency changes significantly during recrystallization. The majority of this shift is due to a dramatic change in texture. Inset: Experimentally measured mode shape before and after annealing.

domly aligned crystallites will exhibit isotropic behavior, $A$ $=1.0$. Conversely, a copper sample with perfectly aligned crystallites will exhibit single crystal behavior, $A=3.2 .{ }^{34}$ The large value of $A$ associated with the recrystallized sample gives a clear indication of the degree of crystallite alignment. Moreover, the large change in $A$ illustrates the dramatic difference between the rolled and annealed textures.

The in situ study involved tracking changes in the RUS spectrum during high temperature processing. For this study the data were taken corresponding to a single predetermined probe position. It is important to note that during the heating and cooling cycles, neighboring modes can intersect in frequency space, increase or decrease in frequency, and attenuate below detectable limits for some portion of the cycle. Thus the question arises, is a single mode tracked or does the tracking process at times follow one or more adjacent modes? One indication that a single mode is tracked is that the mode location is a smooth function of temperature. In addition, because the mode shapes are relatively insensitive to changes in the elastic properties that occur during annealing, ${ }^{35}$ the mode shapes before and after annealing can be used to further support that a single mode was tracked throughout the entire cycle. The in situ mode position for a single mode is shown in Fig. 3. The mode position in frequency space slowly decreases with increasing temperature and then exhibits a rapid and irreversible decrease at approximately $200{ }^{\circ} \mathrm{C}$. Above this transition the mode location continues to decrease in frequency and then upon cooling the mode location increases in frequency. The temperature $\left(\sim 200{ }^{\circ} \mathrm{C}\right)$ at which the mode location suddenly changes corresponds to the recrystallization temperature.

In situ tracking of an isolated mode is complicated for the current case because the experimental mode image at the beginning of the cycle is notably asymmetric. The reason for the asymmetry is that the experimental image is a combination of two closely spaced modes. Experimentally, these two modes cannot be isolated; however, the predicted mode shapes can be combined to form a composite mode. The

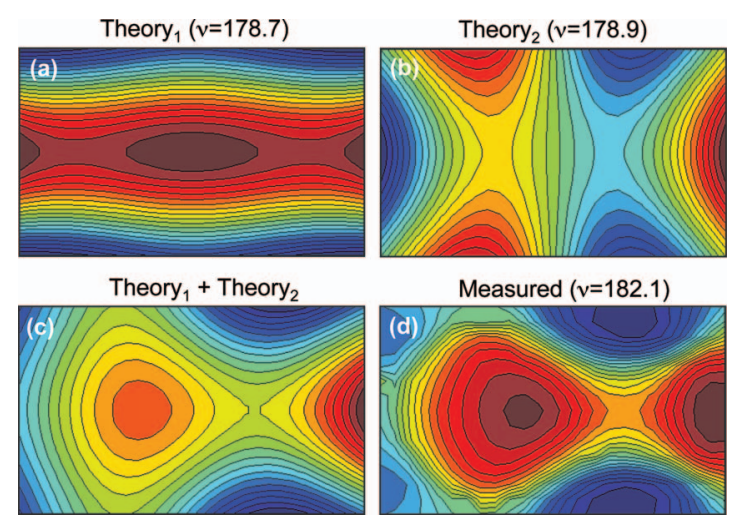

FIG. 4. (Color) [(a) and (b)] The predicted mode shape of two closely spaced modes. (c) The predicted mode shape obtained by summing, with equal weighting, the two predicted modes. (d) Experimentally measured mode shape. Frequencies shown are in $\mathrm{kHz}$.

predicted mode shapes for the modes in question are shown in Figs. 4(a) and 4(b). The composite mode, shown in Fig. 4(c), is obtained by taking the sum of these modes with equal weighting. The experimentally measured mode is shown in Fig. 4(d) for comparison. During the heating cycle, the two closely spaced modes split in frequency and a single mode is tracked for the remainder of the cycle. The experimental mode shape after the heating cycle (lower contour plot in Fig. 3) clearly illustrates that a single mode from the original mode pair was tracked. ${ }^{36}$

Also shown in Fig. 3 is the predicted mode frequency obtained from the EBD data before and after annealing, $178.937 \mathrm{kHz}$ and $145.992 \mathrm{kHz}$ respectively. It is important to note that this large shift in predicted frequency is driven solely by a change in texture. In addition, the predicted frequency shift is comparable to the experimentally measured shift. Thus, for the current case involving the development of a cube texture in heavily deformed $\mathrm{Cu}$, texture driven changes in the elastic character dominate the shift in mode frequency. Dislocation density and pinning length are secondary influences. This analysis illustrates the potential of using EBD data to isolate the influence of texture from that of dislocations.

\section{CONCLUSION}

In situ LRUS was used to monitor microstructure mediated mechanical property evolution in a highly deformed $\mathrm{Cu}$ sample during recrystallization. Resonant mode shapes, determined by raster scanning the interferometric probe before and after annealing, were used to help identify individual modes during high temperature annealing. A detailed discussion of the evolution of a particular mode during annealing was presented. This mode exhibited a shift in center frequency of approximately $20 \%$ of the original value. Using electron backscatter data, it is shown that the majority of this shift is due to changes in texture. Changes in dislocation density and pinning length that occur during annealing are secondary influences. This case exemplifies the capability of LRUS to monitor changes in mechanical properties during high temperature processing. 


\section{APPENDIX: COPPER POLYCRYSTAL AVERAGE ELASTIC STIFFNESS MATRICES}

The elastic constants for single crystal copper are taken as $\hat{C}_{11}=168.4 \mathrm{GPa}, \hat{C}_{12}=121.4 \mathrm{GPa}, \hat{C}_{44}=75.2 \mathrm{GPa}$, and the density ${ }^{34}$ as $\rho=8.96 \mathrm{~g} / \mathrm{cm}^{3}$. Stiffness matrix (in GPA) for the rolled sample is

$$
\left(C_{i j}\right)=\left(\begin{array}{cccccc}
207.2 & 102.0 & 101.5 & 1.3 & 0.7 & 0.8 \\
102.0 & 208.8 & 99.9 & 0.4 & 0.8 & 1.9 \\
101.5 & 99.9 & 209.3 & 0.9 & 1.4 & 1.1 \\
1.34 & 0.4 & 0.9 & 42.0 & 1.0 & 0.7 \\
0.7 & 0.8 & 1.4 & 1.0 & 43.2 & 1.2 \\
0.8 & 1.9 & 1.1 & 0.7 & 1.2 & 43.7
\end{array}\right) .
$$

Stiffness matrix (in GPA) for the annealed sample is

$$
\left(C_{i j}\right)=\left(\begin{array}{cccccc}
174.9 & 118.7 & 117.6 & 0.7 & 0.0 & 0.5 \\
118.7 & 173.7 & 118.8 & 1.7 & 0.2 & 0.3 \\
117.6 & 118.8 & 174.8 & 2.4 & 0.2 & 0.1 \\
0.7 & 1.7 & 2.4 & 68.0 & 0.2 & 0.3 \\
0.0 & 0.2 & 0.2 & 0.2 & 64.9 & 1.0 \\
0.5 & 0.3 & 0.1 & 0.3 & 1.0 & 67.6
\end{array}\right) .
$$

${ }^{1}$ A. Migliori, J. L. Sarrao, W. M. Visscher, T. M. Bell, M. Lei, Z. Fisk, and R. G. Leisure, Physica B 183, 1 (1993).

${ }^{2}$ W. M. Visscher, A. Migliori, T. M. Bell, and R. A. Reinert, J. Acoust. Soc. Am. 90, 2154 (1991).

${ }^{3}$ H. Ledbetter, C. Fortunko, and P. Heyliger, J. Mater. Res. 10, 1352 (1995). ${ }^{4}$ W. Johnson, J. Appl. Phys. 83, 2462 (1998).

${ }^{5}$ J. D. Eshelby, Philos. Trans. R. Soc. London, Ser. A 197, 396 (1949).

${ }^{6}$ J. S. Koehler, in Imperfection in Nearly Perfect Crystals, edited by W. Shockley (Wiley, New York, 1952).

${ }^{7}$ D. O. Thompson and D. K. Holmes, J. Appl. Phys. 27, 713 (1956).

${ }^{8}$ A. V. Granato and K. Lücke, J. Appl. Phys. 27, 583 (1956).

${ }^{9}$ K. Lücke and A. V. Granato, Phys. Rev. B 24, 6991 (1981).

${ }^{10}$ H. M. Simpson and S. J. Kerkhoff, Phys. Rev. Lett. 33, 155 (1974).

${ }^{11}$ H. M. Simpson, A. Sosin, and D. F. Johnson, Phys. Rev. B 5, 1393 (1972)

${ }^{12}$ D. M. Parkin, J. A. Goldstone, H. M. Simpson, and J. M. Hemsky, J. Phys. F: Met. Phys. 17, 577 (1987).

${ }^{13}$ J. N. Lomer and R. J. Taylor, Philos. Mag. 19, 437 (1969).
${ }^{14}$ L. E. Rehn, J. Holder, A. V. Granato, R. R. Coltman, and F. W. Young, Jr., Phys. Rev. B 10, 349 (1974).

${ }^{15}$ W. Benoit, Mater. Sci. Eng., A 370, 12 (2004).

${ }^{16}$ J. Woirgard, J. P. Amiroult, and J. DeFouguet, in Proceedings of Fifth International Conference on Internal Friction and Ultrasonic Attenuation in Solids, edited by D. Lenz and K. Lucke (Springer-Verlag, Berlin, 1975), Vol. 1, p. 392.

${ }^{17}$ P. Gondi, R. Tognato, and E. Evangelista, Phys. Status Solidi A 33, 579 (1976).

${ }^{18}$ N. A. Akhmadeev, N. P. Kobelev, R. R. Mulyukov, Y. M. Soifer, and R. Z. Valiev, Acta Metall. Mater. 41, 1041 (1993).

${ }^{19}$ C. G. Lee and S. Okuda, Phys. Status Solidi A 164, 659 (1997)

${ }^{20}$ K. Foster, S. L. Fairburn, R. G. Leisure, S. Kim, D. Balzar, G. Alers, and H. Ledbetter, J. Acoust. Soc. Am. 105, 2663 (1999).

${ }^{21}$ S. Sato, K. Inagaki, V. E. Gusev, and O. B. Wright, presented at Tenth International Conference in Photoacoustic and Photothermal Phenomena, Rome, Italy (1998), pp. 23-27.

${ }^{22}$ R. S. Schley, K. L. Telschow, J. B. Walter, and D. L. Cottle, Nucl. Technol. 159, 202 (2007)

${ }^{23}$ S. J. Reese, K. L. Telschow, T. M. Lillo, and D. H. Hurley, IEEE Trans. Ultrason. Ferroelectr. Freq. Control 55, 770 (2008).

${ }^{24}$ R. K. Ing and J. P. Monchalin, Appl. Phys. Lett. 59, 3233 (1991).

${ }^{25}$ B. F. Pouet, R. K. Ing, S. Krishnaswamy, and D. Royer, Appl. Phys. Lett. 69, 3782 (1996)

${ }^{26}$ E. F. Lafond, P. H. Brodeur, J. P. Gerhardstein, C. C. Habeger, and K. L. Telschow, Ultrasonics 40, 1019 (2002).

${ }^{27}$ For the in situ test, a $1064 \mathrm{~nm}$ pulsed laser was used for ultrasonic generation and a $532 \mathrm{~nm}$ interferometer, utilizing a bismuth silicon oxide photorefractive element, was used for detection.

${ }^{28}$ W. M. Baldwin, Trans. Am. Inst. Min., Metall. Pet. Eng. 166, 591 (1942).

${ }^{29}$ R. D. Doherty, D. A. Hughes, F. J. Humphreys, J. J. Jonas, D. J. Jensen, M. E. Kassner, W. E. King, T. R. McNelley, H. J. McQueen, and A. D. Rollett, Mater. Sci. Eng., A 238, 219 (1997).

${ }^{30}$ R. Hill, Proc. Phys., Soc. London, Sect. A 65, 349 (1952).

${ }^{31}$ H. M. Ledbetter, J. Phys. D: Appl. Phys. 13, 1879 (1980).

${ }^{32}$ The average grain size for the rolled and annealed samples is 1.5 and $76 \mu \mathrm{m}^{2}$, respectively. For the rolled sample the scanned area is 350 $\times 100 \mu \mathrm{m}$, containing $\sim 647500$ points. The scanned area for the annealed sample is $800 \times 800 \mu \mathrm{m}$, totaling $\sim 740000$ points.

${ }^{33} \mathrm{C}$. Zener, Elasticity and Anelasticity of Metals (University of Chicago Press, Chicago, 1948).

${ }^{34}$ G. W. Farnell, in Physical Acoustics, edited by W. P. Mason and R. N. Thurston (Academic, New York, 1970), Vol. 6, pp. 136.

${ }^{35}$ H. Ogi, K. Sato, T. Asada, and M. Hirao, J. Acoust. Soc. Am. 112, 2553 (2002).

${ }^{36}$ At the end of the heating cycle the center frequencies of the tracked and untracked modes are 143.5 and $141.5 \mathrm{kHz}$, respectively. 